\title{
Dynamic In-situ TEM Investigations of Tribological Interfaces
}

\author{
A.P. Merkle* and L.D. Marks* \\ * Department of Materials Science and Engineering, Northwestern University, 2220 Campus Drive, \\ Cook Hall 2036, Evanston, IL 60208 USA
}

Tribology, the study of surfaces in contact and relative motion, is typically limited by the inability to observe the interface during dynamic measurements of forces. As friction measurements have become increasingly more sensitive due to the development of techniques such as Atomic Force Microscopy (AFM), Nanoindentation, and the Quartz Crystal Microbalance (QCM), many new phenomena have been characterized on the nanoscale that contradict the macroscopic laws of friction. The structure of interfaces and underlying deformation mechanisms such as dislocation drag can help develop a fundamental understanding of the origins of friction between crystalline solids. Furthermore, understanding the behavior of friction at the nanoscale becomes an experimental challenge of precisely identifying the structure and composition of a sliding interface.

An in-situ STM-TEM holder ${ }^{\mathrm{TM}}$ developed by Nanofactory, Inc. was used in a series of investigations of sliding single asperity contacts. The goals of these experiments were to isolate and identify specific structure-friction relationships, including the motion of dislocations and grain boundaries that led to certain sliding conditions. The holder, actuated by a piezoelectric element and capable of local electrical characterization, was used at Argonne National Laboratory in a FEI Tecnai F20ST TEM at $200 \mathrm{kV}$. Characterized samples included amorphous carbon, highly oriented pyrolytic graphite (HOPG), Au islands deposited on HOPG, and Au (001) and (110) film samples. A standard electropolishing technique was used to fabricate the in-situ STM probes (Au and W) with radii of 50 $\mathrm{nm}$ or less.

Graphite is a particularly interesting tribological material due to its low friction and remarkable solid lubricating properties. The shear of weakly bound basal planes should in fact be thought of as stepby-step propagation of dislocations past very weak Peierls barriers [1]. Dienwiebel and coworkers have suggested that graphite-graphite sliding shows a significant dependence on relative lattice orientation [2]. They determined that a graphite flake attached itself to the sliding tungsten probe, thus creating an ideal graphite-graphite sliding interface. Unfortunately, direct evidence of flaking was unattainable. The current in-situ TEM experiments allow for the direct observation and characterization of sliding graphite planes under very similar sliding conditions. In a related study, we sought to observe the graphitization process of amorphous carbon coatings, as confirmed by higher load ex-situ spectroscopy experiments of DLC coatings [3]. The initial results of our experiment indicate that the compliance of the thin amorphous carbon film is too high to generate sufficiently large pressures or temperatures that are necessary to induce a structural transformation to graphite.

$\mathrm{Au}$ (100) and (110) film TEM samples and Au and W STM probes were also used to observe metallic sliding. In particular, we searched for evidence of dislocation motion or lubricated sliding. In order to increase the local contact temperature, the manipulator probe may be biased in order to increase the diffusivity of surface atoms. Sudden structural changes were observed in a small volume of material after applying a bias of 5-7 V for hundreds of milliseconds. After retracting the tip for 
some time, zeroing the bias and approaching again, plastic deformability of the Au was observed. An analogue to the well-known study of liquidlike coalescence of thermally activated Au nanoparticles by Pashley may be suggested from this observation [4]. Of practical importance, this behavior led to the consistent ability to form an ultrasharp Au probe via bias pulsing and pull-off. Figure 1 shows such a probe formed from an $\mathrm{Au}(100)$ sample in contact with a $\mathrm{W}$ tip. Tip radii of less than $5 \mathrm{~nm}$ are routinely achieved by this method and can serve as highly localized electrical, mechanical or topographic probes of nanoparticles and other structures for a variety of applicable studies.

The implementation of a capacitive force sensor within the TEM is necessary for the measurement of friction forces and is the subject of continuing work. By observing sliding single asperity Au contacts inside the TEM, we have been able to plastically deform and fabricate ultrasharp scanning probe tips capable of highly localized topographic and spectroscopic characterization.

\section{References}

[1] R.H. Telling and M.I. Heggie, Phil. Mag. Lett. 83 (2003) 411.

[2] M. Dienwiebel et al., Surface Science 576 (2005) 197.

[3] Y. Liu et al., Surf. \& Coat. Tech. 82 (1996) 48.

[4] D.W. Pashley et al., Philos. Mag. 10 (1964) 127.

[5] This research was carried out in the Electron Microscopy Center at Argonne National

Laboratory, which is supported by the DOE Office of Science under contract \#W-31-109-Eng38.
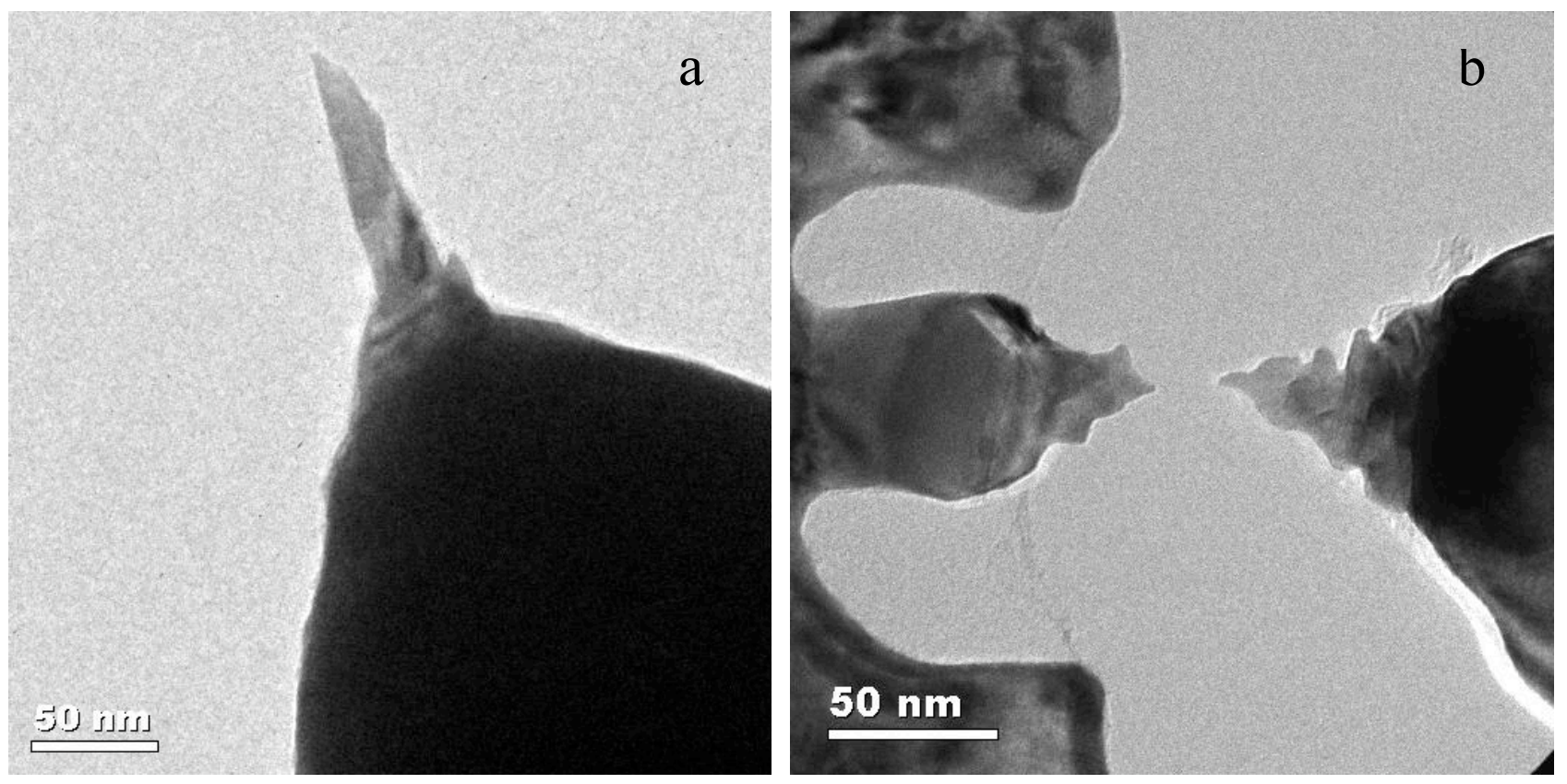

FIG. 1. Bright field TEM images of a tungsten (a) and gold (b) scanning probe shortly after transferring gold from an Au (100) film sample by pulsing 5-7 V for $300 \mathrm{msec}$. Au is transferred to the end of the probe, and is formed to an extremely sharp Au tip by retracting the probe. In-situ fabricated Au tip radii are typically less than $5 \mathrm{~nm}$. 